Teknokultura. Revista de Cultura Digital y Movimientos Sociales

ISSNe: $1549-2230$

http://dx.doi.org/10.5209/TEKN.74375

\title{
Autonomías precarias: un análisis sobre las tecnologías para personas con diversidad funcional intelectual ${ }^{1}$
}

\author{
Joan Moyà-Köhler²; Miquel Domènech ${ }^{3}$
}

Recibido: 18 de febrero de 2021 / Aceptado: 5 de abril de 2021 Open peer reviews

Resumen. Las tecnologías digitales han irrumpido en la vida de las personas con diversidad funcional con la promesa de ofrecer más autonomía y accesibilidad con logros ciertamente remarcables. La diversidad funcional intelectual, sin embargo, ha sido un campo en el que tales desarrollos han sido más modestos y presenta aún ciertas incertidumbres. La cuestión de la autonomía, concretamente, plantea problemas en su puesta en práctica, especialmente cuando se piensa desde la idea de sujeto individual autónomo capaz de tomar decisiones por cuenta propia. A través de un trabajo de carácter etnográfico realizado en un servicio de vida independiente en Cataluña, se lleva a cabo el análisis de dos casos de implantación de tecnología para facilitar la autonomía de personas con diversidad funcional intelectual. A partir de la comparación entre los dos casos, hemos mostrado cómo el resultado final de las experiencias tiene mucho que ver con las interacciones que se dan entre las diferentes concepciones de autonomía que se encuentran presentes y la manera en que se concibe el papel de la tecnología en la consecución de la vida independiente. Pensar toda la red de relaciones que se establece como un conjunto de equilibrios precarios aparece en este trabajo como la clave del éxito que debe permitir lidiar entre asuntos tan complejos como la autonomía, el derecho a decidir y el acompañamiento no invasivo en el día a día de las personas con diversidad funcional.

Palabras clave: diversidad funcional; Estudios de la Ciencia y la Tecnología; tecnología; vida independiente.

\section{[en] Precarious autonomies: an analysis of technologies for people with intellectual disabilities}

Abstract. Digital technologies have burst into the lives of people with disabilities with the promise of offering more autonomy and accessibility with remarkable achievements in some areas. Learning disability, however, has been a field in which such developments have been more modest and still presents certain uncertainties. The issue of autonomy, in particular, poses problems in its implementation, especially when it is considered from the point of view of an autonomous individual subject capable of making independent decisions. Through an ethnographic study carried out in an independent living service in Catalonia, we analyzed two cases of technology implemented to facilitate the autonomy of people with learning disabilities. From the comparison between the two cases, we have shown how the final outcome of the experiences has much to do with the interactions between the different conceptions of autonomy that are present and the way in which the role of technology in achieving independent living is conceived. The study identifies that thinking about the whole network of relationships that is established as a set of precarious balances is critical to dealing with complex matters such as the right to decide, the autonomy or non-invasive support in the daily life of people with learning disabilities.

Keywords: functional diversity; independent living; Science and Technology Studies; technology.

Sumario. 1. Introducción. 2. Diversidad funcional, autonomía, tecnología y ajustes. 3. Metodología. 4. Ajustes y desajustes en las tecnologías para la autonomía. 5. La tecnología y el entramado. 6. Conclusiones. 7. Referencias.

Cómo citar: Moyà-Köhler, J.; Domènech, M. (2021). Autonomías precarias: un análisis sobre las tecnologías para personas con diversidad funcional intelectual. Teknokultura. Revista de Cultura Digital y Movimientos Sociales, 18(2), 127-135. http://dx.doi.org/10.5209/TEKN.74375

\section{Introducción}

Parece una obviedad afirmar que las nuevas tecnologías tienen un papel fundamental en la mejora de las condiciones de vida de las personas con 'diversidad funcional'. Ciertamente los datos corroboran que las nuevas tecnologías se han convertido en instrumentos privilegiados para la facilitación de sus relaciones, su acceso a la información, el disfrute del ocio o su inserción laboral.

\footnotetext{
Beca de Formación Investigadora del programa de la AGAUR de la Generalitat de Catalunya (2014FI_B 01109).

Universitat Oberta de Catalunya/CARENET (España)

E-mail: jmoyak@uoc.edu

3 Universitat Autònoma de Barcelona/Barcelona Science and Technology Studies (STS-b) (España)

E-mail: miquel.domenech@uab.cat
} 
De alguna manera, podría decirse que, en el campo de la discapacidad o la diversidad funcional ${ }^{4}$, las nuevas tecnologías materializan la promesa de la mejora de la autonomía personal y la posibilidad de permanecer en el propio entorno habitual, aunque el acceso a ellas dista aún mucho de ser universal. Si atendemos a los estudios realizados, la mayoría muestran que sigue existiendo una fuerte brecha digital (Goggin, 2016) y que el horizonte de una mayor inclusión del colectivo a través de estas tecnologías está aún lejano (Macdonald y Clayton, 2013). En su informe de 2020, Adecco y Keysight proporcionan algunos datos interesantes al respecto:

$\mathrm{Al}$ ser preguntados directamente por el uso y manejo de las nuevas tecnologías, casi la mitad (un 45\%) declara encontrar barreras. Concretamente, un $42 \%$ declara que le parece 'muy complejo y avanzado su uso', seguido de un $32 \%$ que encuentra problemas de accesibilidad, al no poder manejar ciertos dispositivos debido a in- compatibilidades derivadas de su discapacidad. Por su parte, un $20,6 \%$ no confía en lo digital y 'tiene miedo' a ser engañado y/o víctima de algún fraude, mientras que un $15,9 \%$ afirma carecer de recursos económicos para comprar y adquirir nuevas tecnologías.

También es cierto que en algunas de estas barreras se han hecho avances significativos. La lista de productos que se suelen mencionar para diferentes tipos de diversidad funcional física o sensorial es ciertamente amplia y variada. Ahora bien, cuando se trata de personas con diversidad funcional intelectual, la cosa empeora y se reconoce abiertamente que la variedad es menor, y que ha sido sólo más recientemente que han empezado a aparecer algunas innovaciones como páginas de lectura fácil o apps basadas en pictogramas intuitivos (Fundación Adecco y Keysight Technologies Spain, 2020, p. 13).

Así pues, aunque el desarrollo en relación con las tecnologías digitales ha sido muy destacado estos últimos años en occidente, existen todavía numerosos retos en lo que se refiere a su implementación y uso entre las personas con diversidad funcional, especialmente entre aquellas con diversidad funcional intelectual. Este trabajo se sitúa en el cruce entre la diversidad funcional y los estudios sobre la ciencia y la tecnología. Un cruce especialmente interesante si atendemos a la tensión que históricamente ha vivido el campo de la diversidad funcional en relación con la ciencia y la tecnología y su desarrollo.

El dominio incontestable del paradigma médico sobre la manera en que debe ser concebida la discapacidad (Moser, 2009) encontró una respuesta idiosincrática a través del llamado modelo social de la discapacidad, un modelo que buscó alejarse de la visión tecnológica y cientificista que la visión médica alentó, para focalizarse

\footnotetext{
El concepto de 'discapacidad' es un término que se ha visto contestado esta última década en el estado español. Especialmente a partir de las propuestas del Foro de Vida Independiente que el año 2005 proponía el concepto 'diversidad funcional' como alternativa para rehuir del binarismo normal/anormal o funcional/disfuncional que atraviesa el concepto discapacidad, planteando que la diversidad es inherente al ser humano. Es por ello por lo que en el presente trabajo privilegiaremos el uso del concepto 'diversidad funcional'.
}

en los procesos sociales y culturales. Si bien ello supuso una liberación respecto del corsé biomédico, también es cierto que provocó cierto hermetismo para con otros aportes provenientes de otras disciplinas (Shakespeare, 2013). Es por ello que, para superar ese hermetismo, en este artículo recogemos propuestas que plantean hibridar el modelo social con los Estudios de la Ciencia y la Tecnología (Blume y Galis, 2014). También nos hacemos eco del interés que a partir de los años 90 ha despertado el mundo de la diversidad funcional dentro de los Estudios de la Ciencia y la Tecnología (Galis y Lee, 2015). Estos estudios indagan en los procesos de implantación tecnológica en el campo de la diversidad funcional, especialmente en el campo de la diversidad funcional intelectual, y los factores que contribuyen a su éxito o fracaso.

Para tal fin partimos de un trabajo de carácter etnográfico desarrollado en un servicio de vida independiente para personas con diversidad funcional intelectual. Este trabajo permite vislumbrar algunos elementos interesantes para la reflexión en torno a la articulación de tecnologías pensadas para la autonomía de estas personas. Analizaremos dos casos concretos (uno considerado exitoso y otro fallido) de articulación de tecnologías para mostrar la importancia de tomar en cuenta los factores ecológicos que contextualizan los procesos de implantación. Asimismo, se pondrá de manifiesto la necesidad de atender a los mecanismos que permiten que tales innovaciones tecnológicas permanezcan abiertas al cambio constante y al ajuste continuo (Winance, 2006).

\section{Diversidad funcional, autonomía, tecnología y ajustes}

Antes de analizar los casos analizados, es importante situar de forma rápida algunas ideas y enfoques teóricos que son básicos para entender la manera en que abordamos las complejas relaciones entre diversidad funcional, autonomía y tecnología.

Especialmente importante es situar, aunque sea brevemente, el origen y la manera de articularse, en estos últimos años, del pensamiento social en torno a la diversidad funcional, en particular lo que se conoce como 'modelo social' de la discapacidad. Esta aproximación surge desde los movimientos sociales del ámbito, fundamentalmente del Movimiento de la Vida Independiente, propiciando una identificación colectiva positiva en torno del concepto de 'discapacidad'. De esta manera, aquellas personas que se identifican con el grupo convierten el hecho de compartir una determinada condición en una experiencia colectiva de discriminación (Charlton, 2000), a la que dan sentido a través de la generación de espacios que les permiten redefinir y visibilizar dichas experiencias (Dowse, 2001). Las diferentes luchas promovidas por el Movimiento de la Vida Independiente dieron lugar a algunos principios básicos que se mantienen hasta la fecha: la promoción de los derechos humanos y civiles, la ayuda mutua, el empoderamiento, la responsabilidad de la propia vida, el derecho a asumir riesgos y la vida comunitaria (Lobato, 2018). 
El Movimiento de Vida Independiente experimentaría una rápida expansión y es en Europa, especialmente en el Reino Unido, donde tomaría forma su desarrollo teórico a través del ya mencionado 'modelo social de la discapacidad'. Decepcionados por el enfoque tradicionalmente restrictivo de las prestaciones sociales, los activistas de UPIAS (Union of the Physically Impaired Against Segregation), un grupo no muy numeroso pero influyente, desarrollarían a mediados de la década de los 70 la distinción conceptual entre lo biológico (impairment) y lo social (disability), presentando la 'discapacidad' como una forma de opresión social. Durante las décadas de los 80 y los 90, la combinación del modelo social y los enfoques de vida independiente sería particularmente influyente, llamando la atención del público sobre la importancia de la autoayuda, los servicios dirigidos por los usuarios y las campañas políticas para lograr cambios legales en el campo de la diversidad funcional (Barnes y Mercer, 2010).

Este movimiento activista fue el comienzo de un cambio de enfoque. La diversidad funcional dejaría de ser vista como tragedia personal (Oliver, 2013), lo cual había llevado a situar el problema en el cuerpo del individuo y a buscar las respuestas en un «ejército de aliados de la medicina» (Finkelstein, 2001). En adelante, la diversidad funcional pasaría a contemplarse desde una mirada más emancipadora (Pie et al., 2012), la cual pondrá el acento en el problema de la exclusión social, ecológica y política que acarrea. Ello llevará a focalizar las reivindicaciones del movimiento en el derecho a tener control sobre la propia vida, esto es, a poder decidir y escoger cómo vivir (Ratzka, 2007; Centeno, Lobato y Romañach, 2008). Estas demandas, a nivel práctico, se han articulado a través de dos grandes espacios de incidencia: por un lado, la acción directa sobre el entorno y, por otro, el acceso a los apoyos necesarios para desarrollarse de forma autónoma (Barnes et al., 1999; Shakespeare y Watson, 2001).

Algunas de estas demandas se han concretado en estos últimos años en el campo académico en diferentes estudios sobre la accesibilidad y el desarrollo de elementos técnicos de apoyo, tanto en relación con la implementación de tecnologías para la promoción de la autonomía (Blanco y Latorre, 2008), como para con las formas de desarrollo de estas (Sánchez-Criado y Rodríguez-Giralt, 2017). Se trata pues de un campo lo suficientemente complejo como para no mirarlo desde una sola perspectiva (Corker y Shakespeare, 2020). De ahí la pertinencia de introducir en el análisis elementos propios de los Estudios de la Ciencia y la Tecnología, especialmente lo que se conoce como la 'teoría del actorred' (Domènech y Tirado, 1998).

Efectivamente, es el enfoque simétrico de esta teoría lo que permite ir más allá de la dicotomía capacidaddiscapacidad que articula el discurso del modelo social y dar un giro más después del giro social que este representa. Como indica Galis:

Este enfoque social inicial de la discapacidad carecía de un vínculo crítico entre las experiencias corporales de la discapacidad, el desarrollo de políticas sociales y la configuración de la tecnología (y el entorno construido) (Galis, 2011, p. 828).

Un enfoque simétrico permite también relacionar esta dicotomía capacidad-discapacidad con todas aquellas otras que forman parte del core-set del pensamiento moderno (naturaleza-sociedad, sujeto-objeto o agenciaestructura) evitando de esta manera la reificación de los dos polos tomados siempre como puntos de partida antitéticos. En este sentido, sería tan inadecuado tomar el par 'capacidad/discapacidad' como dos expresiones posibles de una condición natural que adorna a toda persona como reducir su explicación a meros procesos de construcción social. Como muestran los trabajos de Moser (2006) o Winance (2006), para entender cómo se conforma la autonomía de una persona no podemos partir de su adscripción a uno de los dos polos planteados. Las personas están inmersas siempre en entramados sociotécnicos (donde encontramos cuerpos, gadgets técnicos, elementos protésicos o nuevas ecologías) que permiten la aparición de sujetos autónomos, dotados de una capacidad de agencia que se configura a partir de este ensamblaje de elementos heterogéneos, ninguno de los cuales, por sí solo, puede ser señalado como el último responsable de sus acciones.

De manera bastante contra intuitiva, la teoría del actor-red subvierte la concepción de la agencia como un rasgo que sólo tiene que ver con los humanos y muestra que, con demasiada frecuencia, no es posible discernir dónde termina un elemento $\mathrm{u}$ otro del entramado. Cuando no está claro dónde reside la responsabilidad de una acción específica, porque el número de posibles actores involucrados es considerable, ¿cómo identificar al agente responsable de la acción? Como indica Latour, en tal situación hay que cambiar lo que se entiende por acción:

La acción no es una propiedad atribuible a los humanos, sino a una asociación de actantes [...] Si podemos atribuir papeles provisionales 'de actor' a los actantes es sólo porque los actantes se encuentran inmersos en un proceso de intercambio de competencias, es decir, se están ofreciendo mutuamente nuevas posibilidades, nuevas metas, nuevas funciones (Latour, 2001, p. 87).

Al situar la agencia fuera del individuo y considerarla una propiedad emergente de un entramado, la noción de autonomía adquiere un nuevo sentido que resulta especialmente interesante para el ámbito de la diversidad funcional y la propia concepción de lo que implica una vida independiente. Así, cuando Shakespeare (2013) propone que la diversidad funcional debe ser aprehendida como algo que no se puede aislar dentro de la persona, sino que se encuentra en la relación entre el cuerpo con la diversidad funcional y lo que lo rodea, lo que está haciendo es cuestionar, en la línea de la teoría del actor-red (Naslund y Gardelli, 2013), la posibilidad de establecer una clara distinción apriorística entre las diferentes entidades involucradas en relación con sus propiedades intrínsecas. Todo dependerá del entramado y las relaciones a las que dé lugar. En otras palabras, la agencia se distribuye entre los diferentes actores humanos y no humanos, vinculados en un momento especí- 
fico (Law, 1999), explicando desde aquí cómo las personas devienen hábiles o inhábiles (Moser, 2006). Este enfoque teórico invita a investigar qué elementos de la red, tanto humanos como no humanos (Latour, 1993), participan de este proceso de ingeniería heterogénea (Callon y Law, 1995) que supone la formación de este sujeto autónomo.

Finalmente, entender la autonomía como un efecto emergente de un entramado lleva necesariamente a tener que preguntarse acerca de todo el trabajo que hay que hacer para mantener funcional ese mismo entramado, sostenerlo, hacerlo operativo. En este sentido, Winance (2006) utiliza la idea de ajuste:

A través del ajuste, la persona se transforma y se forma. Se configura lo que es material y emocional, lo que es su cuerpo y su mundo. En primer lugar, a través de este ajuste, surge 'un cuerpo extendido' que es a la vez definido (adopta una forma determinada) y transformable. Este 'cuerpo extendido' se refiere a una materialidad común y a unas percepciones comunes. $Y$ en segundo lugar, el mundo de la persona se transforma, se crean posibilidades e imposibilidades, se incluyen algunas entidades y se excluyen otras. El ajuste es al mismo tiempo la conformación del cuerpo y la conformación de ese mundo (Winance, 2006, p. 67).

Esta perspectiva implica una idea importante: la durabilidad de la materialidad, su estabilidad, se constituye a través de la solidez de los vínculos, no tiene que ver con la calidad del material en sí. Para Winance (2006), el acto de ajustar pone de manifiesto que el vínculo es la afectación de los elementos en juego, no sólo su interrelación, lo cual resulta crucial aquí para pensar en el uso de tecnologías para facilitar la autonomía. Desde esta perspectiva, cada nueva incorporación no debe tomarse como la simple adjunción de un elemento más, de un actor más, sino que es preciso contemplar los vínculos que va a establecer con los otros elementos del entramado y los efectos que estos van a producir en relación a la persona con diversidad funcional.

\section{Metodología}

Los dos casos en los que se basa este artículo son tomados del trabajo de inspiración etnográfica, o etnografía focalizada (Knoblauch, 2005), realizado en el marco de la tesis doctoral de uno de los autores del texto en un servicio de apoyos personales directos para la Vida independiente para personas con diversidad funcional intelectual, el 'Me'n vaig a casa' de la Fundació Catalana de la Síndrome de Down.

Este trabajo etnográfico tuvo lugar entre los años 2015 y 2016, y estaba compuesto por el seguimiento de 11 unidades de convivencia. Durante estos períodos se llevó a cabo una observación participante durante el acompañamiento a las personas de apoyo en el día a día del servicio y se participó de las distintas reuniones de conformación y de seguimiento de los casos, así como con las familias u otras personas de referencia. El proceso quedó recogido en un diario de campo en el que se anotaban todos aquellos elementos que se disponían en el día a día de las usuarias desde el servicio.

Para el trabajo se han seleccionado dos de estos once casos, aquí debidamente anonimizados, que permiten dar cuenta de dos momentos en los que desde el servicio se trabaja en la articulación de elementos tecnológicos para con la autonomía de las usuarias.

Tabla. 1. Tabla de participantes. Fuente: elaboración propia

\begin{tabular}{|c|c|c|c|c|c|}
\hline Usuarias & $\begin{array}{c}\text { Número de encuentros } \\
\text { etnográficos }\end{array}$ & Edad & Procedencia & $\begin{array}{c}\text { Unidad de } \\
\text { convivencia }\end{array}$ & $\begin{array}{c}\text { Tiempo en el } \\
\text { servicio }\end{array}$ \\
\hline Manoli & 8 & $\begin{array}{c}\text { Apenas comienza la } \\
\text { treintena }\end{array}$ & $\begin{array}{c}\text { Vivir sola, sin } \\
\text { apoyos }\end{array}$ & Sola & Un año \\
\hline Vanessa y Francesca & 20 & $\begin{array}{c}\text { Ambas rondan la } \\
\text { treintena }\end{array}$ & $\begin{array}{c}\text { Las dos de su casa } \\
\text { familiar }\end{array}$ & Amigas & Cuatro años \\
\hline
\end{tabular}

Aparte de la observación directa durante esos meses, se llevaron a cabo dos entrevistas a cada usuaria, así como a las familias o personas de referencia de estos y a las personas que les brindaban apoyo. Las entrevistas fueron debidamente transcritas y analizadas mediante la técnica del análisis temático.

La información de las entrevistas, junto a los datos obtenidos durante el proceso de confección del diario de campo, acabaron dando lugar a lo que algunos autores definen como una descripción densa (Geertz, 2000): una especulación elaborada que emerge a partir de las interacciones y significados situados en el propio campo.

\section{Ajustes y desajustes en las tecnologías para la autonomía}

El servicio 'Me'n vaig a casa' de la Fundació Catalana de la Síndrome de Down es pionero a nivel estatal y ha servido de inspiración para muchos otros proyectos puestos en marcha desde las asociaciones del sector. Se basa en los postulados de los servicios supported living (Hagner y Klein, 2005) que aparece en los EE.UU. como respuesta al traumático cierre de las instituciones de clausura que se vivió en los años 80 bajo el mandato de Ronald Reagan (Smull y Danehey, 1994). A partir de una planificación individualizada basada en el apoyo 
y la asistencia personal a personas con diversidad funcional intelectual, estos servicios buscan lograr la máxima inclusión dentro de la comunidad, facilitando a las personas que lo deseen las herramientas necesarias para poder desarrollar un proyecto de vida propio fuera del hogar familiar (Ruf, 2007).

A nivel práctico, esto implica que las personas pueden escoger de qué manera y con qué personas quieren vivir. Para ello, los profesionales del servicio llevan a cabo dos tipos de estrategias. Por un lado, realizan reuniones con el círculo de confianza, es decir, personas elegidas por las propias usuarias para tomar de forma colegida las decisiones; generalmente se trata de familiares. Por otro lado, elaboran, conjuntamente con las personas usuarias, el llamado Plan de Atención Personal. Se trata de un plan de acción centrado en la persona que sirve de guía para el servicio a la hora de articular qué apoyos son necesarios, así como para fijar objetivos en el desarrollo de la vida independiente de la usuaria.

En su despliegue, el servicio de vida independiente cuenta con un conjunto de apoyos socio-materiales. Dispone, por ejemplo, de un determinado número de horas semanales de acompañamiento por parte de una persona de apoyo - un máximo de diez-que se financian con una ayuda directa de la Generalitat de Catalunya a la vida independiente. Además, hay todo un entramado de elementos técnicos que se ponen a disposición de las personas usuarias. Estos pueden ir desde elementos analógicos -como carteras diferentes con el dinero justo para determinadas actividades, cajas fuertes para la gestión del dinero o calendarios para marcar los ritmos diarios- hasta elementos más sofisticados -por ejemplo, un conjunto de códigos QR repartidos por la casa que dan acceso a vídeos tutoriales para el uso de determinados gadgets-.

Tales apoyos permiten a la persona con diversidad funcional desarrollarse de forma autónoma en un ensamblaje heterogéneo de elementos que operan, como también se verá en los ejemplos, a través de la necesidad de conciliar distintos discursos en relación con lo que debe ser 'la vida independiente'.

\subsection{Un caso de desajuste}

El primer apunte etnográfico que queremos recuperar es un pequeño episodio que vivimos con Vanessa y Francesca. Se trata de dos usuarias con síndrome de Down que vivían en su propio hogar en un barrio cerca de la Ronda de Dalt en Barcelona.

Vanessa no trabajaba, Francesca sí lo hacía a media jornada. Llevaban viviendo cerca de cuatro años en el piso, por el que se encontraban repartidos un conjunto de códigos QR. Podían verse al lado del televisor, en el armario de las ollas de la cocina, en la puerta del microondas o al lado del termostato. Durante la observación, sin embargo, no se pudo apreciar que hicieran uso de los mismos.

En una de las entrevistas se le preguntó a la persona de apoyo que las acompañaba unas horas al día acerca de la utilidad de esos códigos. Explicó que eran códigos para acceder a vídeos tutoriales que habían realizado a través de una compañía tecnológica para que las usuarias pudieran acceder a instrucciones de uso de los diferentes elementos que se disponían en la casa:

Sí, los usamos al inicio... bueno ellas. Y claro, bien. Era una prueba piloto. Ahora la verdad es que lo usan poco porque ya no hace falta, cuando ya lo han usado un par de veces ya está, ¿no? (...) Claro, ellas ahora ya lo saben usar y no miran el vídeo, pero bueno, ahí siguen los códigos.

Estos códigos se disponían en relación con distintos elementos y pretendían facilitar que las usuarias ganaran autonomía, pudieran hacer uso por su cuenta de los distintos utensilios de la casa o electrodomésticos. El acceso a los vídeos debería facilitar hacerlo de forma adecuada, esto es, 'segura'. La tecnología pretendía situarse en medio de la tensión entre, por un lado, la voluntad de delegar a las usuarias las tareas del día a día y, por el otro, la necesidad del servicio de minimizar posibles riesgos para con su integridad (Entrevista Laura, noviembre 2016).

Con la delegación en los QR, en teoría, las usuarias no tenían que preguntar cada vez a la persona de apoyo y conseguían usar ellas mismas los diferentes aparatos y ganar autonomía. Parecía un elemento ideal, pero no. En realidad, no acabó de funcionar. En parte porque, una vez aprendido el uso, no hacía falta ver de nuevo el vídeo. En parte, también, porque las necesidades, al cabo de un tiempo, pasaban a ser otras. Los códigos se volvían obsoletos muy rápidamente $\mathrm{y}$, de hecho, ni siquiera las propias usuarias eran ya conscientes de su presencia, por lo cual, contactaban telefónicamente con las personas de apoyo antes que hacer uso de aquellos.

\subsection{Un caso de ajuste}

El segundo caso es algo menos sofisticado, pero resulta muy ilustrativo. Manoli es una mujer con diversidad funcional intelectual que vive sola en un piso con una gran terraza llena de plantas en medio de la ciudad de Barcelona. Trabaja a media jornada.

Aquí, la gestión del dinero es parte de lo que el servicio llama 'un problema', como podemos ver en el siguiente extracto de entrevista:

Yo probé de vivir sola primero, pero mis padres no lo veían demasiado bien, soy "su niña". A pesar de eso hicimos la prueba y fue una cagada... porque tenía acceso a la tarjeta de crédito, y... (...) no me sabía controlar, no? Mi punto flojo es el tema dinero. Ambos intentos fueron mal y dije a mi padre que buscáramos una fundación porque se me iba la mano (Entrevista Manoli, septiembre 2015).

Manoli conoce el valor y puede usarlo por su cuenta, pero si tiene acceso a su cuenta bancaria de forma directa puede gastar grandes cantidades en lo que en su círculo de confianza se consideran 'caprichos'. Esto lleva al servicio a trabajar sobre ello y restringirle el acceso. Una restricción que lleva a Manoli a reivindicar su deseo de gastar un dinero que, por otro lado, ha ganado ella. 
La reclamación de un uso no restringido de su dinero es una expresión de su deseo de autonomía, al mismo tiempo que es fuente de preocupación de la familia. $\mathrm{Y}$ es en este punto que el servicio debe tomar un papel para conseguir una solución a esta tensión, lo cual hace a partir de dos estrategias. Por un lado, a través de disponer para Manoli de diferentes monederos con distintas cantidades de dinero para diferentes actividades, además de facilitarle una tarjeta de crédito con un tope para los días de la compra. Es decir, a través de un conjunto de gadgets se permite a Manoli administrar su propio dinero. Todo ello queda dentro de lo acordado en las reuniones de círculo con personas de confianza que mencionábamos anteriormente. Por otro lado, el servicio le facilita también una cierta cantidad de dinero extra para que se lo pueda gastar, por ejemplo, en el café de la máquina del trabajo. El servicio permite, por tanto, un uso secreto de pequeñas cantidades con la aprobación de la persona de apoyo, aunque este uso no se considere adecuado, de forma similar a lo que Goffman (1972) define como 'ajustes secundarios'. Se permite, pues, un espacio de asociaciones ambiguas e identidades múltiples donde la figura de la persona de apoyo emerge como indispensable para ajustar y mantener el ensamblaje en acción.

El servicio necesita encontrar un orden que no pasa por la aprobación de los padres acerca de ese uso particular del dinero. Un orden basado en esta permisividad controlada del gasto de pequeñas cantidades a espaldas de los padres de Manoli que permite mantener el funcionamiento del entramado. Una dinámica, sin embargo, que debe mantener un cierto equilibrio precario y, por lo tanto, quedar circunscrito a pequeñas cantidades de dinero, que no representen un peligro para la relación con la familia. Cada solución propuesta, en general, se satura rápidamente, por lo que es necesario volver a negociar una nueva forma del entramado que sea funcional; un nuevo proceso de negociación en busca de un nuevo orden. Un orden, pues, surgido dentro de un espacio de constante tira y afloja.

Este arreglo, sin embargo, no se sitúa en una balanza. No consiste en encontrar un punto medio entre mucho y poco dinero para Manoli. Tampoco se arregla con la opción de dejarle llevar mucho o de no llevar nada. Como hemos visto, la disposición del servicio no es tan simple, es el producto de negociaciones y conexiones que a menudo se alejan del punto de partida.

\section{La tecnología y el entramado}

La primera de las reflexiones que podemos destacar, atendiendo a lo presentado hasta el momento, se refiere a la singularidad del campo en el que nos encontramos. $\mathrm{Y}$ es que la diversidad funcional intelectual es un espacio especialmente interesante para reflexionar en torno a la autonomía.

En el campo de la diversidad funcional las principales propuestas que articulan la noción de autonomía derivan de los trabajos realizados en torno, o desde, las personas con diversidad funcional física. En el caso del activismo en el estado español, por ejemplo, desde el Foro de Vida Independiente se ha trabajado la autonomía a partir de la idea de la asistencia personal, entendida esta como un apoyo que debe llevar a cabo las tareas que la persona con diversidad funcional le indica, actuando como «sus brazos, sus oídos, sus ojos, etc.» (Centeno, Lobato y Romañach, 2008). De este modo, la autonomía queda ligada de forma inexorable a la capacidad de tomar decisiones:

Una persona que tiene una tetraplejia, como es el caso del autor de este texto, puede no ser autónomo a la hora de realizar muchas tareas $y$, sin embargo, es plenamente capaz de tomar decisiones. La confusión de estos dos conceptos ha tenido como consecuencia la institucionalización de personas con poca autonomía física y plena autonomía moral, que se han visto así privadas de su capacidad de llevar una vida en igualdad de oportunidades, para la que estaban plenamente preparados (Romañach, 2012, p. 49).

En este escenario, las personas con diversidad funcional intelectual ponen en tensión algunos de los presupuestos sobre los que se construye la idea de 'autonomía'. Si la autonomía es una cuestión de toma de decisiones, cuando esta llega a un espacio en el que la capacidad de tomar 'buenas decisiones' está discutida, como sucede con la diversidad funcional, se hace evidente un límite para esta manera de concebirla. Y en gran medida este límite y las formas en las que se piensan las tecnologías para la vida independiente en el caso de la diversidad funcional intelectual, se ponen de manifiesto en los dos casos que acabamos de presentar.

El primer ejemplo, de Vanessa y Francesca, muestra cómo la capacidad de acción de una tecnología impone un guion de uso (script) muy específico (Akrich, 1992) en el que el individuo es autónomo en la medida en que, de forma disciplinada, sigue el curso de acción permitido. La implantación de códigos QR, efectivamente, las vuelve autónomas respecto a sus personas de apoyo, pero sólo hasta cierto punto. Sólo si entendemos la autonomía como una acción llevada a cabo sin una persona de apoyo. Ahora bien, si asumimos la autonomía como la capacidad de decidir, parece que la disposición sociotécnica ha sido diseñada, precisamente, para evitar tomar decisiones. Los códigos QR están ahí para ser usados en todas las ocasiones, sin que se espere que las usuarias decidan cuándo llamar y cuándo no. En este caso la tecnología parece estar pensada desde el prejuicio que plantea que las personas con diversidad funcional intelectual carecen de la capacidad para tomar decisiones.

La autonomía es considerada, pues, como un ejercicio individual y no desde la apertura de posibilidades de relación. Los códigos QR son un intento fallido de substituir a la persona de apoyo por un recurso tecnológico (Latour, 2017) que debería proporcionar las mismas instrucciones que aquella podría ofrecer presencialmente. Dado que la posibilidad de una relación diferente no está contemplada, cuando algo no sale bien se pone de manifiesto que se trata de un modelo cerrado que colapsa y deja de ser útil. De esta manera, podríamos decir que la autonomía de las usuarias se manifiesta en su práctica de 
llamar a la persona de apoyo y prescindir del código QR que han decidido que no les es útil. Sería otro tipo de autonomía, algo así como lo que Winance (2016) califica como raw autonomy (o autonomía en bruto), que aparece en el momento en que una persona con diversidad funcional es recalcitrante ante lo que se espera de ella y actúa en contra del guion preestablecido. Una autonomía que emerge de forma inesperada, en ese acto de no hacer lo que se espera.

De este modo, lo que hemos podido ver en este primer caso es que no basta con tener buenas tecnologías, ni siquiera que estas estén bien diseñadas o sean accesibles. No se trata de cómo es este actor concreto, de cuáles son sus características, sino de su papel en el entramado, de cómo se vincula con el resto de elementos, de los afectos que producen o los efectos que tienen en la configuración de la persona con diversidad funcional como persona autónoma desde el ideal que performa la tecnología.

Tan interesante es comprender por qué la disposición sociotécnica del primer caso falla, como desentrañar por qué funciona la disposición de los elementos en el entramado que presentamos en el segundo. En este caso, las tecnologías que entran en juego, algo más rudimentarias, encuentran con éxito su incorporación a la ecología que está dispuesta en el ensamblaje concreto, y consigue establecer vínculos fuertes con el resto de los elementos. $\mathrm{Su}$ funcionalidad radica, aunque parezca sorprendente, en hacer posible que dos nociones de autonomía incompatibles puedan coexistir en un mismo entramado (la de los padres - para los cuales la autonomía consiste en 'vivir sola', pero de forma segura, limitando las decisiones que Manoli puede tomar- y la de la propia Manoli -para la cual, la autonomía consiste en tomar sus propias decisiones). Y al hacerlo, hacen posible que surja una nueva noción de autonomía que asume su carácter relacional, abierto, cambiable, precario, en definitiva.

En este caso podemos ver cómo el servicio trabaja desde la capacidad de adaptación, de cambio, de ser 'hakeable', elementos que se tornan atributos indispensables para su articulación. Esto es, estas tecnologías (tarjetas de crédito con tope, diferentes monederos, tiques de la compra...) tienen la capacidad de facilitar equilibrios precarios y concretos, si es necesario traicionando partes de esta red y las relaciones que se han establecido allí (Singleton y Michael, 1998).

Así, de una manera tan precaria e inestable, es la forma en la que el servicio logra ser funcional y encontrar un 'equilibrio estacionario' entre muchos. A través de un complejo ejercicio de traducción de los diferentes actores, trabajando en sus procesos de 'interesamiento' (López, 2012), ajustando constantemente los elementos socio-técnicos que componen este ensamblaje, lejos de resolver la tensión entre las diferentes nociones de autonomía que circulan, el servicio las mantiene. Y aquí recae el éxito de esta propuesta: la autonomía no se piensa aquí desde la imposición de un modo de ser autónomo, individual, sino desde la posibilidad de relacionarse y cambiar, desde la provisionalidad de estas relaciones que se establecen.
Vemos en el segundo caso cómo estas distintas nociones de autonomía que hemos visto, aparentemente incompatibles, están presentes constantemente, pero no funcionando alternativamente, ni una encima de la otra, ni siquiera en paralelo, sino que el servicio encuentra el ajuste, la sincronía necesaria que permite la coexistencia de ambas, lejos del equilibrio, en la tensión (MoyàKöhler, 2018). O más precisamente, buscando un orden en esa misma tensión, porque como hemos visto, es precisamente a partir de la existencia de la tensión que el servicio puede trabajar, operando como una 'estructura disipativa' (Prigonie y Stengers, 1983).

Tomamos la noción de estructura disipativa del campo de la química y los estudios de sistemas complejos porque nos permite entender bien cómo las tecnologías que se incorporan en la ecología concreta que dispone el servicio de vida independiente consiguen asentarse en posiciones inestables, fuera del equilibrio prefijado de antemano. En relación con el dinero, ya hemos visto que no se trata de dar mucho o nada, como tampoco de dar justo la mitad. Se trata, casi, de dar y no dar a la vez, de situarse en un modo que permita un orden allá donde no era imaginable: lejos del equilibrio.

En palabras de Prigogine y Stengers (1983) estas estructuras «son islas de orden en un mar de desorden». De hecho, según las propias autoras, estas estructuras aumentan su orden a expensas de un creciente desorden del medio. Necesitan desorden para existir y estabilizarse precariamente a través de una 'entrada de energía' que deriva del sistema original. La entrada constante de energía, esta tensión en la que se mueven estos espacios, es lo que explica el éxito de las tecnologías que se sitúan en ella, porque deben ser lo suficientemente duras para mantenerse, pero lo suficientemente amoldables para soportar los cambios. Para Prigogine y Stengers (1983) este 'equilibrio estacionario' solo es posible si, lejos del orden, las estructuras resultantes son precarias, a la vez que coherentes y autoorganizadas.

En definitiva, lo que hemos mostrado en cada ejemplo es la manera en que una determinada concepción de autonomía se articula con una disposición sociotécnica. En el primer caso, la autonomía se concibe como la actuación de unas usuarias que funcionan solas. Para ello, se disponen elementos tecnológicos cuya función es sustituir a las personas de apoyo. Unos dispositivos que quedan ahí, sin más, 'abandonados'. En este caso, los dispositivos tecnológicos parecen concebidos como meros intermediarios (Domènech y Tirado, 1998) que harán sin mayor dificultad una función de acompañamiento. Por el contrario, en el segundo caso la autonomía antes que un rasgo personal es un efecto emergente del entramado del que la usuaria forma parte. No es posible, entonces, pensar la tecnología como mera intermediaria, dispuesta siempre a ejecutar ciegamente unas instrucciones predeterminadas. Vista así la autonomía, las relaciones que la usuaria establece con todo el entramado de elementos del que forma parte son indispensables y deben ser el punto de partida para pensar cualquier implantación tecnológica. En este sentido, asumir la precariedad de la disposición sociotécnica, lleva a considerar como esencial todas las tareas de cuidado y 
ajuste que constantemente toda infraestructura necesita (Denis y Pontille, 2014; Sánchez-Criado et al., 2014), un trabajo sobre los elementos materiales y los elementos emocionales en juego (Winance, 2006) que termina haciendo posible este ensamblaje que es «la usuaria dentro del servicio, actuando».

\section{Conclusiones}

A lo largo de este artículo hemos podido mostrar los efectos que tiene no prestar atención a la ecología de relaciones en la que todo dispositivo tecnológico se inscribe. Lejos de poder ser concebidos como meros dispositivos de plug-n-play, hemos de asumir que los artefactos van a afectar y ser afectados, van a establecer relaciones con sus usuarias y con el resto de los elementos que conforman el entramado en el que se introducen. Es por ello por lo que hemos señalado la importancia de pensar en el entramado. No para configurar una disposición de elementos que se mantenga firme y sólida para llevar a cabo su función, sino, por el contrario, posibilitar disposiciones que necesitan de la precariedad para funcionar. Por ello, hemos llamado a tales disposiciones estructuras 'disipativas', asumiendo que su vigencia es siempre temporal, provisional y que, para que se mantengan durante un cierto tiempo, es preciso que estén abiertas al constante ajuste y reajuste, a encontrar soluciones no desde el orden, sino desde el desorden.

Pensar toda la red de relaciones que se establece como un conjunto de equilibrios precarios aparece en este trabajo como la clave del éxito que debe permitir lidiar entre asuntos tan complejos como la autonomía, el derecho a decidir y el acompañamiento no invasivo en el día a día de las personas con diversidad funcional.

\section{Referencias}

Akrich, M. (1992). The de-scription of technical objects. En Shaping Technology/Building Society. Studies in Sociotechnical Change (W. Bijker y J. Law, pp. 205-224). Cambridge, MA: MIT.

Barnes, C. y Mercer, G. (2010). Exploring Disability: A Sociological Introduction. Cambridge: Polity Press.

Blanco, F. J. y Latorre, M. J. (2008). Promoción de la autonomía en situaciones de dependencia: el uso de nuevas tecnologías. Docencia e Investigación: Revista de la Escuela Universitaria de Magisterio de Toledo, 33(18), 1.

Blume, S. y Galis, V. (2014). Introduction: STS and Disability. Science, Technology \& Human Values, 39(1), 98-104. https://doi. org/10.1177/0162243913513643

Callon, M., y Law, J. (1995). Agency and the hybrid «Collectif». The South Atlantic Quarterly, 94(2), 481-507.

Centeno, A., Lobato, M., y Romañach, J. (2008). Indicadores de Vida Independiente (IVI) para la evaluación de politicas y acciones desarrolladas bajo la filosofia de Vida Independiente. Recuperado de: https://studylib.es/doc/7216923/indicadoresde-vida-independiente-ivi-para-la-evaluació...

Charlton, J. (2000). Nothing about Us without Us. Berkeley: University of California Press.

Corker, M. y Shakespeare, T. (2002) Mapping the terrain. En Disability/postmodernity: embodying disability theory (M. Corker y T. Shakespeare, pp. 1-17). Londres: Continuum.

Denis, J. y Pontille, D. (2014). Material Ordering and the Care of Things. Science, Technology \& Human Values, 34, 1-30. https:// doi.org/10.1177/0162243914553129

Domènech, M. y Tirado, F, (1998). Sociología simétrica. Ensayos sobre ciencia, tecnología y sociedad. Barcelona: Gedisa.

Dowse, L. (2001). Contesting Practices, Challenging Codes: Self advocacy, disability politics and the social model. Disability \& Society, 16(1), 123-141. https://doi.org/10.1080/713662036

Finkelstein, V. (2001). The social model of disability repossessed. Manchester Coalition of Disabled People, 1, 1-5.

Fundación Adecco y Keysight Technologies Spain (2020). Tecnología y Discapacidad. Recuperado de: https://fundacionadecco. org/wp-content/uploads/2019/07/Descarga-informe-tecnolog\%C3\%ADa-y-discapacidad-2020.pdf

Galis, V. (2011). Enacting disability: how can science and technology studies inform disability studies? Disability \& Society, 26, 825-838. https://doi.org/10.1080/09687599.2011.618737

Galis, V. y Lee, F. (2015). A Sociology of Treason: The Construction of Weakness, Science, Technology, \& Human Values 39(1), 154-179. https://doi.org/10.1177/0162243913512681

Geertz, C. (2000). La interpretación de las culturas. Barcelona: Gedisa.

Goffman, E. (1972). Internados: Ensayos sobre la situación social de los enfermos mentales. Buenos Aires: Amorrortu.

Goggin, G. (2016). Disability and digital inequalities: Rethinking digital divides with disability theory. En Theorising Digital Devides. (M. Ragnedda y G. Muschert). London: Routledge. Recuperado de: https://ses.library.usyd.edu.au/bitstream/handle /2123/15844/2017Goggindisability\%26digitaldivide.pdf?sequence=4\&isAllowed=y

Hagner, D. y Klein, J. (2005). Home ownership for individuals with disabilities: factors in mortgage decisions. Journal of Disability Policy Studies, 15(4), 194-200. https://doi.org/10.1177/10442073050150040101

Knoblauch, H. (2005). Focused Ethnography. Forum Qualitative Sozialforschung/Forum: Qualitative Social Research, 6(3). https://doi.org/10.17169/fqs-6.3.20

Latour, B. (1993). Nunca hemos sido modernos. Madrid: Debate

Latour, B. (2001). La esperanza de Pandora. Ensayos sobre la realidad de los estudios de la ciencia. Barcelona: Gedisa

Law, J. (1999). Political Philosophy and Disabled Specificities. (Centre for Science Studies Lancaster University). Recuperado de: https://www.lancaster.ac.uk/fass/resources/sociology-online-papers/papers/law-political-philosophy-and-disabilities.pdf

Lobato, M. (2018). Movimiento Vida Independiente. (Vida Independiente Comunidad Valenciana). Recuperado de http://vicoval. org/movimiento-de-vida-independiente/

López, D. (2012). Transiciones hacia otra(s) teoría(s) del actor-red: agnosticismo, interés y cuidado. En Teoría Del Actor-Red. Más Allá de los Estudios de Ciencia y Tecnología, (F. Tirado y D. López, pp. 157-187). Barcelona: Amentia. 
Macdonald, S. J., y Clayton, J. (2013). Back to the future, disability and the digital divide. Disability \& Society, 28(5), $702-718$.

Moser, I. (2006). On Becoming Disabled and Articulating Alternatives. Cultural Studies, 19(6), 667-700. https://doi. org/10.1080/09502380500365648

Moser, I. (2009). A body that matters? The role of embodiment in the recomposition of life after a road traffic accident. Scandinavian Journal of Disability Research, 11(2), 83-99. https://doi.org/10.1080/15017410902830520

Moyà-Köhler, J. (2018). Sincronitzant autonomies: estudi d'un servei de vida independent per a persones amb la sindrome de Down. (Tesis doctoral, Universitat Autònoma de Barcelona). Recuperado de: https://ddd.uab.cat/pub/tesis/2018/ hdl_10803_666756/jmk1de1.pdf

Naslund, R., y Gardelli, A. (2013). "I Know, I Can, I Will Try": Youths and Adults with Intellectual Disabilities in Sweden using Information and Communication Technology in their everyday life. Disability \& Society, 28(1), 28-40. https://doi.org/10.10 $80 / 09687599.2012 .695528$

Oliver, M. (2013). The social model of disability: thirty years on. Disability \& Society, 28(7), 1024-1026. https://doi.org/10.108 $0 / 09687599.2013 .818773$

Pié, A. (2012). Introducción. En Deconstruyendo la dependencia: Propuestas para una vida independiente (A. Pié, pp. 21-46). Barcelona: Editorial UOC.

Prigogine, I., y Stengers, I. (1983). La nueva alianza: metamorfosis de la ciencia. Madrid: Alianza Universidad.

Ratzka, A. (2007). Independent Living for people with disabilities: from patient to citizen and customer. (Gladnet Collection). Recuperado de: https://ecommons.cornell.edu/bitstream/handle/1813/76679/Adolf_Ratzka people_with_disabilities_from_patient_to_citizen_customer.pdf?sequence $=1 \&$ isAllowed $=\mathrm{y}$

Romañach, J. (2012) Ética y derechos en la práctica diaria de la atención a la dependencia: autonomía moral vs. autonomía física. En Deconstruyendo la dependencia: Propuestas para una vida independiente (A. Pié, pp. 37-59). Barcelona: Editorial UOC.

Ruf, J. (2007). Independent Living and Social Inclusion. International Medical Review on Down Syndrome, 11(3), 40-43. https:// doi.org/10.1016/S2171-9748(07)70056-9.

Sánchez-Criado, T., López, D., Roberts, C. y Domènech, M. (2014). Installing Telecare, Installing Users : Felicity Conditions for the Instauration of Usership. Science, Technology \& Human Values, 39(5), 694-719. https://doi.org/10.1177/0162243913517011

Sánchez-Criado, T. y Rodríguez-Giralt, I. (2017). Caring through design? En torno a la silla and the 'joint problem-making'of technical aids. En Care and Design: Bodies, Buildings, Cities (C. Bates, R. Imrie y K. Kullman, pp.198-218). London: Wiley.

Shakespeare, T. y Watson, N. (2001). The social model of disability: An outdated ideology? En Exploring Theories and Expanding Methodologies: Where we are and where we need to go. (Research in Social Science and Disability, Vol. 2). (S. Barnartt y B. Altman, pp. 9-28). Bingley: Emerald Group Publishing Limited. https://doi.org/10.1016/S1479-3547(01)80018-X

Singleton, V. y Michael, M. (1998). Actores-red y ambivalencia. Los médicos de familia en el programa británico de citología de cribaje. En Sociología simétrica. Ensayos sobre ciencia, tecnología y sociedad (M. Domènech y F. J. Tirado, pp. 171-217). Barcelona: Gedisa.

Smull, M. W. y Danehey, A. J. (1994). Increasing quality while reducing costs: The challenge of the 1990s. En Creating Individual Supports for People with Developmental Disabilities (V. Bradley, J. Ashbaugh y B. Blaney, pp. 197-211). Baltimore: Paul Hall Brookes.

Winance, M. (2006). Trying out the Wheelchair: The Mutual Shaping of People and Devices through Adjustment. Science, Technology, \& Human Values, 31(1), 52-72. https://doi.org/10.1177/0162243905280023

Winance, M. (2016). Rethinking disability: Lessons from the past, questions for the future. Contributions and limits of the social model, the sociology of science and technology, and the ethics of care. Alter, 10(2), 99-110. https://doi.org/10.1016/j. alter.2016.02.005 